\title{
Water Soluble Luminescent Cyclometalated Platinum(II) Complex - A Suitable Probe for Bio-imaging Applications
}

Sheik Saleem Pasha ${ }^{\mathrm{a} \#}$, Pradip Das ${ }^{\mathrm{b} \#}$, Nigam P. Rath ${ }^{\mathrm{c}}$, Debashree Bandyopadhyay ${ }^{\mathrm{d}}$, Nikhil R. Jana $^{* \mathrm{~b}}$, Inamur Rahaman Laskar*a

${ }^{a}$ Department of Chemistry, Birla Institute of Technology and Science, Pilani Campus, Pilani, Rajasthan, 333031 India, ir_laskar@pilani.bits-pilani.ac.in; ${ }^{\mathrm{b}}$ Centre for Advanced Materials, Indian Association for the Cultivation Science, Jadaavpur, Kolkata 32, India camnrj@iacs.res.in; 'Department of Chemistry and Biochemistry, University of Missouri-St Louis, University Blvd, St Louis, MO 63121, USA, rathn@umsl.edu; ${ }^{\mathrm{d}}$ Department of Biology, Birla Institute of Technolgy and Science, Hyderabad Campus, Hyderabad, Telangana, 500078, India, banerjee.debi@hyderabad.bits-pilani.ac.in

${ }^{\#}$ Both authors have the same contribution to this work

Keywords: Platinum complex, Bio-imaging, Luminescence, Toxicity.

\section{Abstract}

A highly water soluble platinum(II) complex, $[\mathrm{Pt}(\mathrm{ppy})(\mathrm{en})] \mathrm{Cl}$, (where ppy = 2phenylpyridine and en = ethane-1, 2-diamine) with a strong green luminescence in solution was synthesized in two steps. Initially, an intermediate complex, $[\mathrm{Pt}(\mathrm{ppy})(\mathrm{ppyH})(\mathrm{Cl})], 1$ was isolated which was transformed into the light-emitting mononuclear platinum(II) complex, [Pt(ppy)(en)]Cl, 2. The green emission of $\mathbf{2}$ was changed into yellow in their solid state. The strong $\pi^{\cdots} \pi$ intermolecular interactions that interplays between the phenyl rings lying into the two consecutive molecules which led to the solid state emission. The low cytotoxicity of 2 ensures the feasibility for using these in bioimaging probe. The testing bioimaging results demonstrate that the compound penetrates the cell membrane in both cancer (HeLa, U87MG and Nuro2a) and normal cells (CHO-K1 and 3T3-L1). The cellular uptake was observed to much higher in cancerous cells only. Co-localization study confirmed the localization of it into the cell nucleus.

Bio-imaging is used to label the cells and cell organelles to understand the mechanistic pathways of biochemical reactions [1]. The most desirable criteria for bio-imaging probes has a good thermal stability [2], water solubility [3], low cyto-toxicity [4], high cellular 
uptake [4] and selectivity for specific organelles [4]. Till date many types of bio-imaging luminescent probes like organic luminogens [4], quantum dots [4b, 5] and heavy metal based cyclometalated complexes [6] etc have been reported. Among these luminophores, heavy metal [Ir(III), Pt(II), Os(IV), Re(I) and Rh(III)] based complexes have received a special attention. These probes are superior in terms of phosphorescent emission at room temperature [7], higher stability [7], larger quantum yield [8], lower photo bleaching effect [8] and greater Stoke's shift [9] in comparison with other bio-imaging probes.

Platinum(II) is a $\mathrm{d}^{8}$ system, preferably a 4-coordinated square planar geometry. In contrast, the $\mathrm{d}^{6}$ metal ion complexes show distorted octahedral geometry. The aggregation property plays a significant role in self-assembling of non-biological entities into living systems [10]. The square planar geometry could be helpful in the formation of self assembly or stacking [11]. The stacking of Pt(II) complex may result in higher emission quantum yields, longer excited state lifetimes and reduced reactivity as compared to the single component motifs [10]. One of the prime challenges in utilizing such complexes as probe molecule in bioimaging is water solubility. Very few water soluble Platinum(II) complexes have been reported as bio-imaging probes e.g., M. H. W. Law et.al have reported an amphiphilic and water-soluble platinum(II) complex $\left[\mathrm{Pt}\left(\mathrm{C}^{\wedge} \mathrm{N} \wedge \mathrm{N}-\mathrm{C}_{18}\right)\left(\mathrm{P}\left\{\mathrm{C}_{6} \mathrm{H}_{4}-\mathrm{SO}_{3}\right\}_{3}\right)\right]^{2-}$ as a biological probe [12]. This complex was successfully localized in the plasma membrane of HeLa cell. M. Selke et al have reported [13] a Platinum(II) complex [Pt(Thpy)(HThpy)py]ClO 4 which was applied for nucleus staining and labelling of mitochondria with an $\mathrm{IC}_{50}$ of $3.29 \mu \mathrm{M}$ for this complex. L. Mishra et. al have also reported [14] [ $\left.\{\mathrm{Pt}(\mathrm{en}) \mathrm{L}\}_{2}\right] \mathrm{PF}_{6}$, where $\mathrm{LH}_{2}$ is $\mathrm{N}, \mathrm{N}-$ bis(salicylidene)-p-phenylenediamine and en is 1,2-diamino ethane for nucleus staining. However, this complex is highly toxic $\left(\mathrm{IC}_{50}=11.5 \mu \mathrm{M}\right)$. Che and co-workers have described [15] Pt(II)-allenylidene complex as nucleus staining, but it is also toxic $\left(\mathrm{IC}_{50}=19\right.$ $\mathrm{mM}$ ). Recently, we have reported [7] $\mathrm{C}^{\wedge} \mathrm{NPtPP}[\mathrm{C} \wedge \mathrm{N}=2$-phenlpyridine; $\mathrm{PP}=$ bis(diphenylphosphino)ethane)] complex which stains on the nucleus but it shows the $\mathrm{IC}_{50}$ around $10 \mu \mathrm{m}$ and not water soluble. Herein, we report a water soluble, strongly luminescent and low toxic [Pt(ppy)(en)]Cl complex as an efficient nuclear bioimaging probe. 
The complex 2 was synthesized in two steps (Scheme 1). It was resulted through chelation of ethylenediamine to platinum(II) by replacing two ligands $\left(\mathrm{Cl}^{-}\right.$and $\mathrm{N}$-bonded phenylpyridine) from the metal. The structure of the complex $\mathbf{2}$ was determined by NMR spectroscopy and X-ray single crystal structure analysis (Fig. 1). ${ }^{1} \mathrm{H}$ NMR spectra of complex 2 showed aromatic proton signals in the range, $\delta=7.1-8.7 \mathrm{ppm}(\mathrm{S} 1 \dagger)$. The four protons of $\left(\mathrm{CH}_{2}-\mathrm{CH}_{2}\right)$ of ethylenediamine ligand were observed as multiplets at $\delta=2.67 \mathrm{ppm}$ and the four N-H protons of ethylenediamine appear in the range, $\delta=5.38-6.12 \mathrm{ppm}(\mathbf{S 1} \dagger) .{ }^{13} \mathrm{C}$ NMR spectra of complex 2 showed aromatic carbons signals in the range $\delta=119-168 \mathrm{ppm}$. The two carbons of $\left(\mathrm{CH}_{2}-\mathrm{CH}_{2}\right)$ of the ethylenediamine ligand were observed at $\delta=48.3$ and $44.0 \mathrm{ppm}$ (S2†). Complex 2 exhibits a green emission in the solution which is changed into a bright yellow emission in the solid state (Fig. 2). The packing diagram of the complex (Fig. 1) clearly indicates the presence of $\pi-\pi$ stacking which is being interplayed in between the phenyl rings of consecutive molecules. It shows a green emission in water with $\lambda_{\max } 510 \mathrm{~nm}$ under excitation of $375 \mathrm{~nm}$ (Fig. 3) and a yellow emission in the solid state with $\lambda_{\max } 553 \mathrm{~nm}$ with exciting at $400 \mathrm{~nm}$ (Fig. 4). This observation suggests that a new excited state i.e., ${ }^{3} \mathrm{MLLCT}$ is formed from the existing ${ }^{3} \mathrm{MLCT}$ state (vide supra, molecular modelling) when the molecules gets assembled in solid state and the energy of the new state gets stabilized. As a result, the energy of the solid state of the complex gets lowered [green (solution) $\rightarrow$ Yellow (solid)].

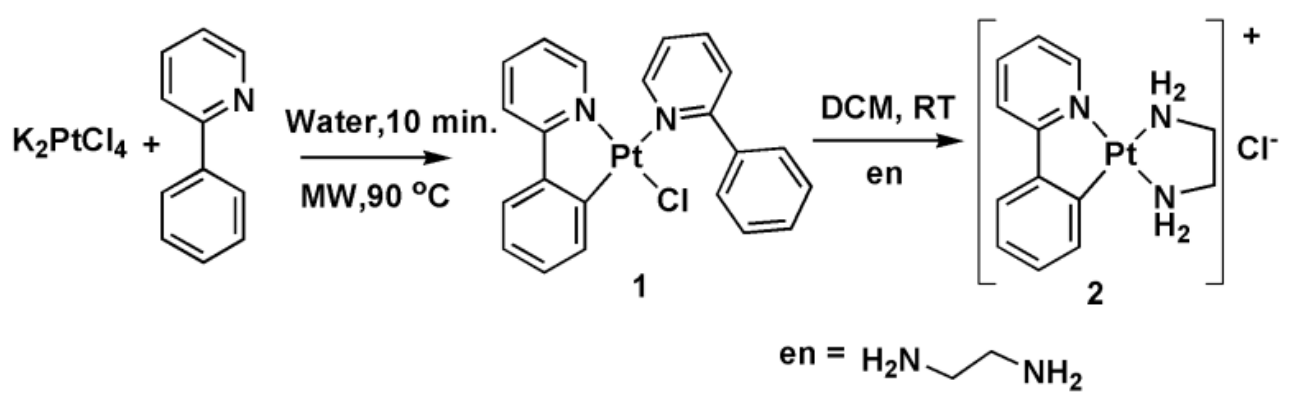

Scheme 1. Synthetic route and chemical structures of complexes $\mathbf{1}$ and $\mathbf{2}$. 


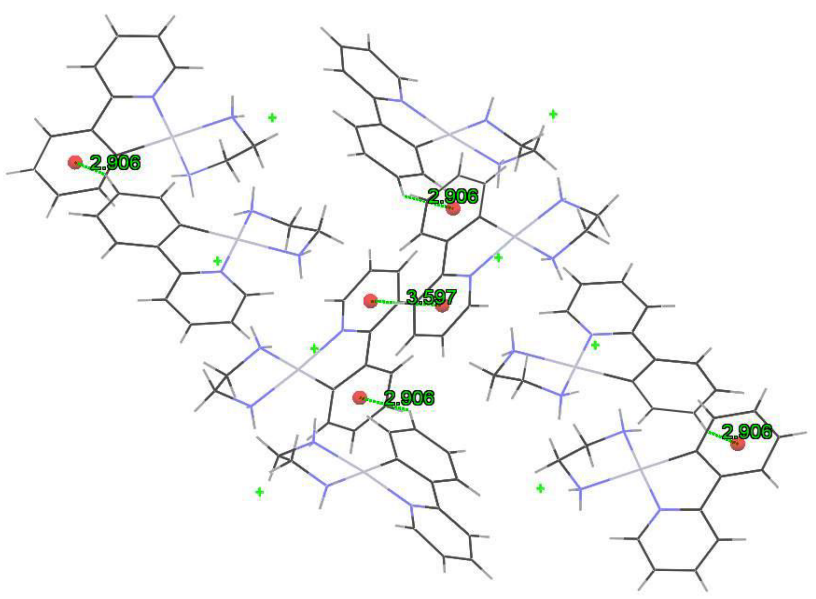

Fig. 1. Crystal packing diagram of complex 2 showing a $\pi \cdots \cdots \pi$ type stacking interactions between pyridyl rings (3.597 $\AA$ ) and between $\mathrm{C}-\mathrm{H} \pi$ (phenyl) to centroid of adjacent phenyl ring (2.906 $\AA)$.

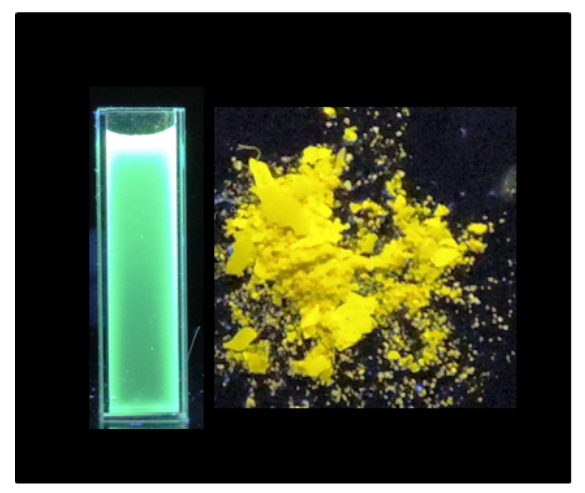

Fig. 2. Luminescent images of complex 2 under UV excitation at $365 \mathrm{~nm}$; solution (water, left); solid (right). 


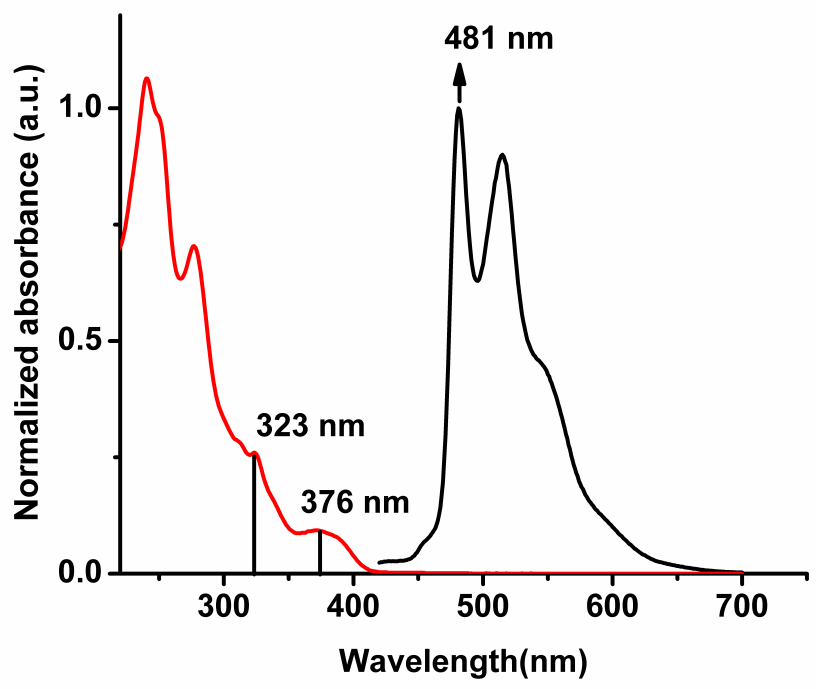

Fig. 3. UV-Visible absorption (left) and photoluminescence spectra (right) of complex 2 in $1 \times 10^{-5} \mathrm{M} \mathrm{MeOH}$.

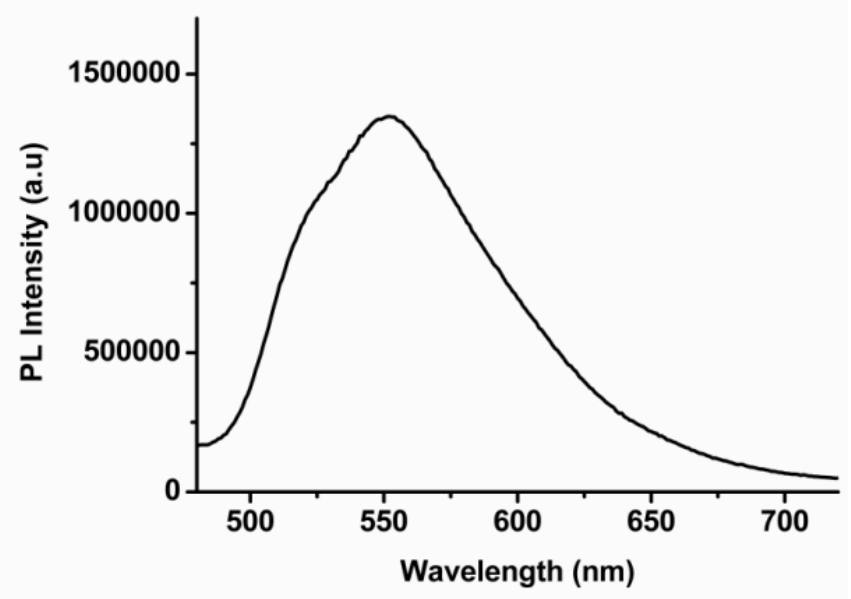

Fig. 4. Solid state photoluminescence spectra of complex 2.

DFT and TD-DFT Study: Single electron excitation with TD-DFT has produced ten different excitations from $S_{0}-S_{1}$ state to $S_{0}-S_{10}$ states. First three excitations are reported (Table S1 $\dagger$ ). The first and third excitation $\left(\mathrm{S}_{0}-\mathrm{S}_{1}, \mathrm{~S}_{0}-\mathrm{S}_{3}\right)$ wavelengths (371 nm and $306 \mathrm{~nm}$, respectively) obtained from TD-DFT calculations matched well with the experimental excitation wavelengths in UVvisible range (376 nm and $323 \mathrm{~nm}$, respectively). The oscillator strengths for these two transitions are higher as compared to $\mathrm{S}_{0}-\mathrm{S}_{2}$ (Table $\mathbf{S 1} \dagger$ ) which indicates that these two transitions 
are more probable than the other. $\mathrm{S}_{0}-\mathrm{S}_{1}$ transitions mainly involve HOMO and LUMO orbitals (Table S1†). HOMO has a significant contribution from the platinum(II) ion and the pheyl part of the 2-phenylpyridine (Fig. 5); whereas, LUMO purely lies on the fused ring, co-ordinated to platinum(II). Thus, $\mathrm{S}_{0}-\mathrm{S}_{1}$ transition has a significant metal to ligand charge transfer (MLCT) character (Table S1†). Ground state singlet to excited state triplet transition $\left(\mathrm{S}_{0}-\mathrm{T}_{1}\right)$ has a calculated wavelength of $483 \mathrm{~nm}$. Although, this transition is spin-forbidden, it is observed as a consequence of inter-system crossing. The $\mathrm{S}_{0}-\mathrm{T}_{1}$ transition wavelength obtained from TD-DFT calculation which is in agreement with the experimental maximum emission wavelength of 481 $\mathrm{nm}$. This transition also involves the HOMO and LUMO orbitals, however to the lesser extent as compared to $S_{0}-S_{1}$ transition. That reflects the MLCT character observed in $S_{0}-T_{1}$ transition (Table S2†).

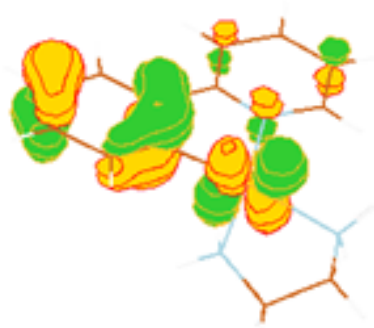

HOMO

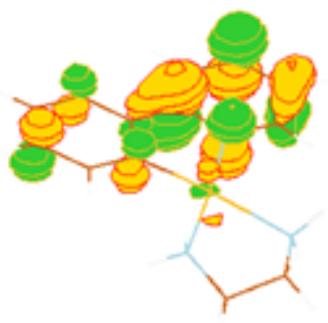

LUMO

Fig. 5. Frontier Molecular Orbitals for complex 2. (Calculations are performed by GAMESS US software; for visualization of the molecular orbital, MOLDEN software was used).

The highly luminescent water soluble platinum(II) complex, 2 has been used as a bioimaging probe. The cellular uptake study shows that $\mathbf{2}$ enters into both cancer and normal cells. However, the uptake is high in cancer cells as compared to normal cells (Fig. 6 and S3†). Time dependence study shows that uptake of complex 2 increases with incubation time and gradually localizes into cell nucleus (S4† and $\mathbf{S 5} \dagger$ ). The fluorescence imaging study at different incubation time ranging from 15 min to 24 hrs shows that the complex 2 is localized in cytoplasm in the first one hour and then slowly localizes into the cell nucleus within 2 hrs (S4† and S5 $†$ ). The colocalization study shows that the complex 2 predominantly co-localizes with nucleus staining dye with longer time of incubation (Fig. 7). Luminescence intensity profile of the complex 2 and 
propidium iodide labeled HeLa cells further indicates the staining of the cell nucleus by the complex 2 (S6†). The complex 2 binds to nucleic acids of the nucleus possibly due to the intercalation with the nucleic acids stabilized by the $\pi-\pi$ stacking interactions between the nucleic acid bases and aromatic ligand of complex [20].

The cellular toxicity of complex 2 towards the cancer cells (HeLa, U87MG and Nuro2a) and normal cells (CHO-K1 and 3T3-L1) has been determined by MTT assay. Fig. 8 shows that cell viabilities were $>80 \%$ in presence of the tested concentration of complex 2 (in the range of 20$170 \mu \mathrm{M})$. The result indicates that complex 2 has low cytotoxicity in the labeling concentration range.
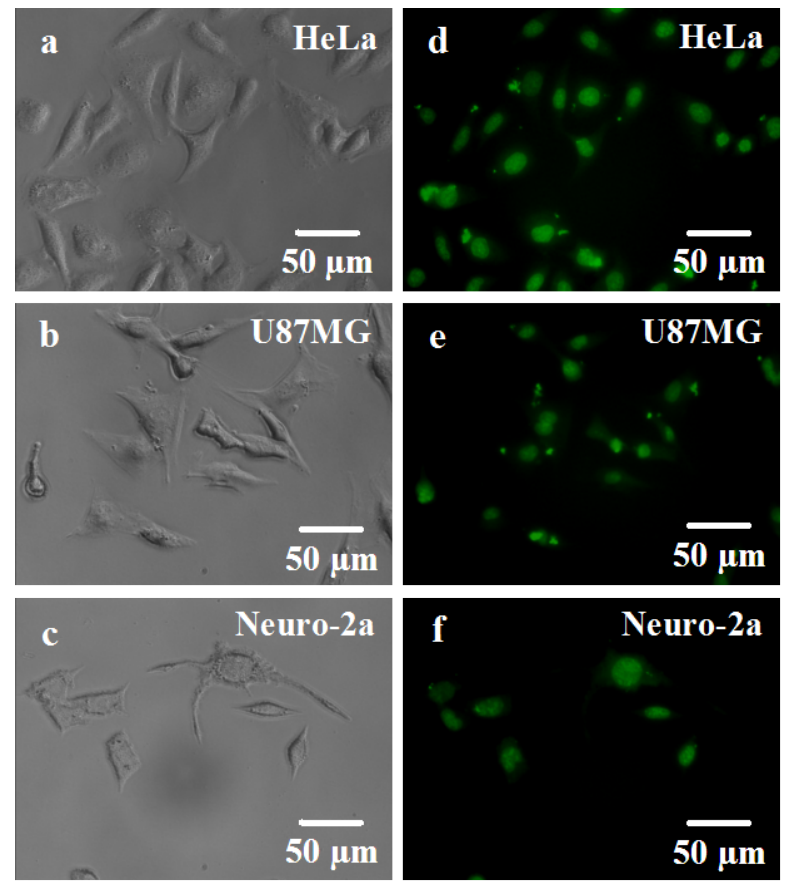

Fig. 6. Differential interference contrast images (a-c) and luminescence images (d-f) of complex 2 labeled HeLa, U87MG and Neuro-2a cells. Cells are incubated with complex 2(final concentration of $170 \mu \mathrm{M}$ ) for 4 hrs and then washed cells are imaged under UV excitation. 

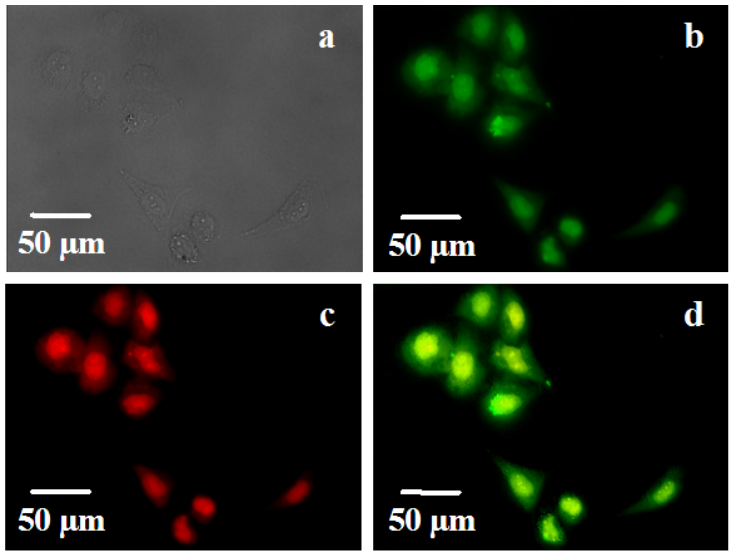

Fig. 7. Co-localization study of complex 2 and propidium iodide labeled HeLa cells. Fixed HeLa cells are imaged under differential interference contrast mode (a), UV excitation for imaging of complex 2 (b), green excitation for imaging of propidium iodide that label cell nucleus (c). Merged image of b and c shows significant co-localization of complex $\mathbf{2}$ and propidium iodide (d).

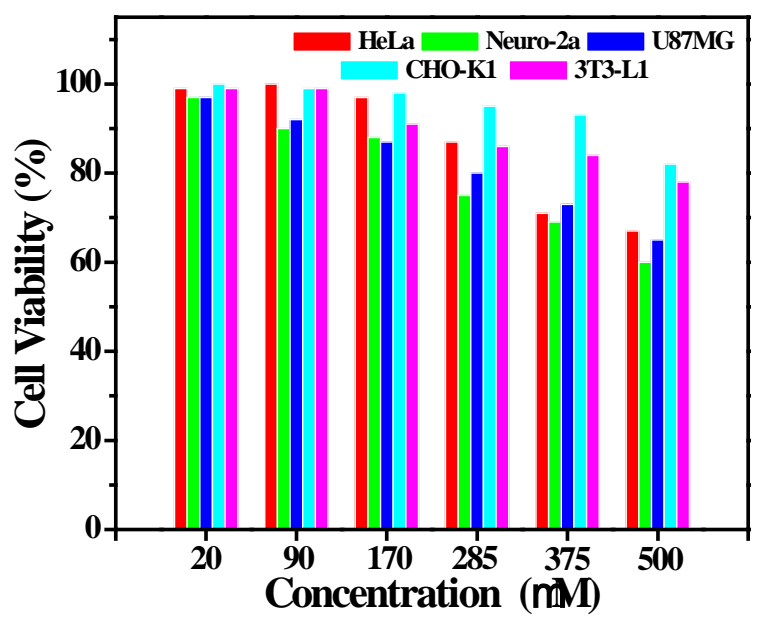

Fig. 8. Viability of different cells in presence of complex 2 of different concentrations. Cells are incubated with complex 2 of different concentrations for 24 hrs and then cell viability is determined assuming 100 \% viability for control sample having no complex 2. 
We have measured the stability of complex 2 in pure fetal bovine serum (FBS) similar to plasma up to one week. The digital images and photoluminescence spectra of complex 2 shows the complex has high colloidal stability (S7†). We have studied the Z-stacking fluorescence imaging of complex 2 labeled HeLa cells to confirm the nucleus accumulation of complex. The Zstacking images show that the most of the complex $\mathbf{2}$ is localized in cell nucleus (S8†).

A water soluble Platinum(II) based complex was synthesized by a simple methodology. The solid state emission of the complex is different from its solution state which arises due to the formation of a new electronic state, ${ }^{3}$ MLLCT. This complex spontaneously enters into cell cytoplasm and then localize preferably into cell nucleus. The cellular uptake of $\mathbf{2}$ by cancerous cells was observed higher as compared to the normal cells. Low cytotoxicity of this complex facilitates for the possible application as bio-imaging probe.

\section{Acknowledgements}

We thank the 'Department of Science and Technology (DST), Govt. of India' (No. SB/S1/IC13/2014) and 'Council of Scientific and Industrial Research (CSIR), Govt of India' No. 01/2551/12/EMR-II for the financial support. 'UGC-SAP' and 'DST-FIST' are acknowledged for their instrumental support to Department of Chemistry, BITS Pilani. PD acknowledges CSIR India for providing research fellowship. Funding from the National Science Foundation, USA (MRI, CHE-0420497) for the purchase of the ApexII diffractometer is acknowledged.

\section{References}

[1] (a) D.Ling, W. Park, S. J. Park, Y. Lu, K. S. Kim Chem. Soc. Rev. 2015, DOI:10.1039/C4Cs00275J; (b) Z. Guo, S. Park, J.Yoon, I. Shin, Chem. Soc. Rev. 43 (2014) 16; (c) Q. Zhao, C. Huang and Fuyou L, Chem. Soc. Rev. 40 (2011) 2508.

[2] J. R. Lacowicz, Springer, Principle of Fluorescence Spectroscopy, New York, 2006.

[3] (a) Y. Xia, L. Dong, Y. Jin, S. Wang, L Yan, S. Yin, S. Zhou and B. Song, J. Mater Chem. B, 3 (2015) 491; (b) S. Sahu, B. Behera, T. K. Maiti and S. Mohapatra, Chem. Commun. 48 (2012) 8835; (c) V. F. Moreira, Flora L. T. Greenwood and M. P. Coogan, Chem. Commun. 46 (2010) 186. 
[4] (a) A. J. Amoroso and S. J. A. Pope, Chem Soc. Rev. 2015, DOI 10.1039/C4CS00293H; (b) A. Chakraborty and N. R. Jana, J. Phys Chem C. 119 (2015) 2888; (c) X. Sun, X. Huang, J. Guo, W. Zhu, Y. Ding, G. Niu, A. Wang, G. O. Kiesewetter, Z. L. Wang, S. Sun, X. Chen, J. Am. Chem. Soc. 136 (2014) 1706; (d) H. Kobayashi, M. Ogawa, R. Alford, P. L. Choyke and Y. Urano, Chem. Rev. 110 (2010) 2620.

[5] (a) X. Michalet, F. F. Pinaud, L. A. Bentolila, J. M. Tsay, S. Doose, J. J. Li, G. Sundaresan, A. M. Wu, S. S. Gambhir and S. Weiss, Scienc. 307 (2005) 538; b) J. Zheng, P. R. Nicovich and R. M. Dickson, Annu. Rev. Phys.Chem. 58 (2007) 409.

[6] (a) M. X. Yu, F. Y. Li, Z.G. Chen, H. Hu, C. Zhan, H. Yang and C. H. Huang, Anal. Chem. 81 (2009) 930; (b) H. Hu, M. X. Yu, F. Y. Li, Z. G. Chen, X. Gao, L. Q. Xiong and C. H. Huang, Chem.Mater. 20 (2008) 7003; (c) L. Q. Xiong, T. S.Yang, Y. Yang, C. J. Xu and F. Y. Li, Biomaterials. 31 (2010) 7078; (d) Z. G. Chen, H. L. Chen, H. Hu, M. X. Yu, F. Y. Li, Q. Zhang, Z. G. Zhou, T. Yi and C. H. Huang, J. Am. Chem. Soc. 130 (2008) 3023; (e) J. Zhou, M. X. Yu, Y. Sun, X. Z. Zhang, X. J. Zhu, Z. H. Wu, D. M. Wu and F. Y. Li, Biomaterial. 32 (2011) 1148; (f) Q. Liu, C. Y. Li, T. S. Yang, T. Yi and F. Y. Li, Chem. Commun. 46 (2010) 5551; (g) F. Wang and X. G. Liu, Chem. Soc. Rev. 38 (2009) 976; (h) F. Wang, D. Banerjee, Y. Liu, X. Chen and X. G. Liu, Analyst. 135 (2010) 1839.

[7] S. S. Pasha, P. Alam, S. Dash, G. Kaur, D. Banerjee, R. Chowdhury, N. Rath, A. R Choudhury and I. R. Laskar, RSC Advances. 4 (2014) 50549.

[8] (a) P. Alam, M. Karanam, A. R. Choudhury and I. R. Laskar, Dalton Trans. 41 (2012) 9276. (b) P. Alam, P. Das, C. Climentc, M. Karanam, D.Casanovac, A. R. Choudhury, P. Alemany, N. R. Jana and I. R. Laskar, J. Mater. Chem C. 2 (2014) 5618.

[9] T. D. wang and G. Triadafilopoulos, Gastrointestinal Endoscopy. 61 (2005) 686.

[10] M. Mauro, A. Aliprandi, D. Septiadi, N. S. Kehr and L. D. Cola, Chem. Soc. Rev. 43 (2014) 4144.

[11] (a) V. H. Houlding and V. M. Miskowski, Coord. Chem. Rev. 111 (1991) 145; (b) V. M. Miskowski and V.H. Houlding, Inorg. Chem. 30 (1991) 4446; (c) C. A. Strassert, M. Mauro and L. De Cola, Adv.Inorg. Chem. 63 (2011) 48; (d) I. M. Sluch, A. J. Miranda, O. 
Elbjeirami, M. A. Omary and L. M. Slaughter, Inorg. Chem. 51 (2012) 10728; (e) B. Ma, J. Li, P. I. Djurovich, M. Yousufuddin, R. Bau and M. E. Thompson, J. Am. Chem. Soc. 127 (2005) 28; (f) D. Kim and J. L. Bre'das, J. Am. Chem. Soc. 131 (2009) 11371; (g) M. Kato, C. Kosuge, K. Morii, J. S. Ahn, H. Kitagawa, T. Mitani, M. Matsushita, T. Kato, S. Yano and M. Kimura, Inorg. Chem. 38 (1999) 1638.

[12] C. K. Koo, K. L. Wong, C. W. Y. Man, H. L. Tam, S. W. Tsao, K. W. Cheah and M. H. W. Lam, Inorg. Chem. 48 (2009) 7501.

[13] S. W. Lai, Y. Liu, D. Zhang, B. Wang, C. N. Lok, C. M. Che and M. Selke, Photochem. Photobiol. 86 (2010) 1414.

[14] N. Kumari, B. K. Maurya, R. K. Koiri, S. K. Trigun, S. Saripella, M. P. Coogan and L. Mishra, Med.Chem.Comm. 2 (2011) 1208.

[15] X. S. Xiao, W. L. Kwong, X. Guan, C. Yang, W. Lu and C. M. Che, Chem. Eur. J. 19 (2013) 9457.

[16] Nardelli, M. J. Appl. Crystallogr. 28 (1995) 569.

[17] R. Sangeetha Gowda K, R. Blessy Baby Mathew, C. N. Sudhamani, H. S. Bhojya Naik, Biomedicine and Biotechnology. 2 (2014) 1. 


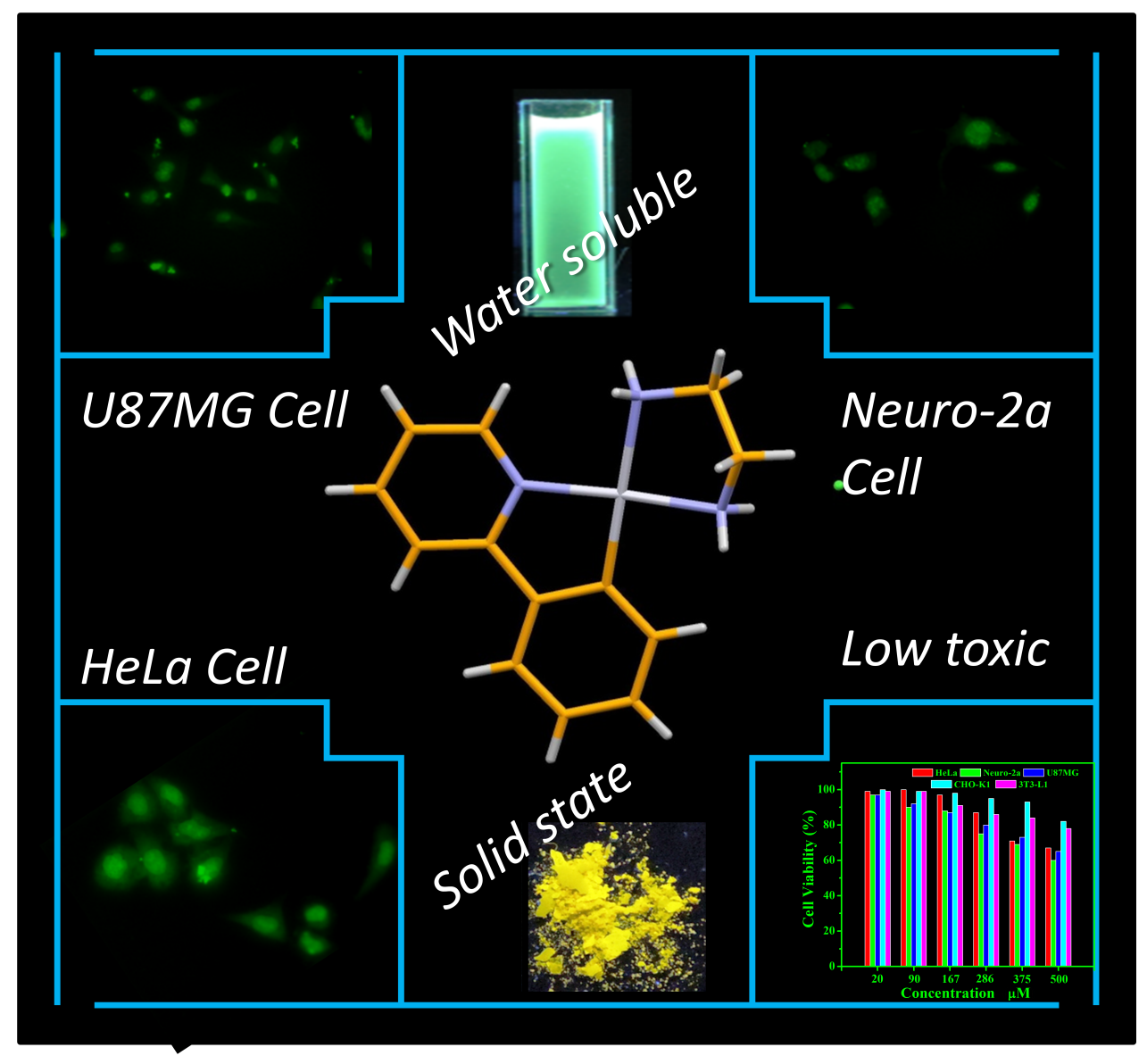

Graphical Abstract 